\title{
Caméra aux poings. Images dans la grève : des usages pluriels dans la lutte syndicale
}

Camera in Hand: A Variety of Uses in the Trade Union Struggle

Jordane Burnot et Lina Cardenas

\section{OpenEdition}

\section{Journals}

Édition électronique

URL : https://journals.openedition.org/itti/2321

DOI : 10.4000/itti.2321

\section{Éditeur}

Université de Poitiers

\section{Référence électronique}

Jordane Burnot et Lina Cardenas, «Caméra aux poings. Images dans la grève : des usages pluriels dans la lutte syndicale », Images du travail, travail des images [En ligne], 12 | 2022, mis en ligne le 22 février 2022, consulté le 01 avril 2022. URL : http://journals.openedition.org/itti/2321 ; DOI : https:// doi.org/10.4000/itti.2321

Ce document a été généré automatiquement le 1 avril 2022.

Images du travail, travail des images 


\section{Caméra aux poings. Images dans la grève : des usages pluriels dans la lutte syndicale}

Camera in Hand: A Variety of Uses in the Trade Union Struggle

Jordane Burnot et Lina Cardenas

1 Le mardi 2 avril 2019 - suite à des élections professionnelles victorieuses - des salariées de Pizzorno (groupe de ramassage d'ordures ménagères) du site de Vénissieux se mettent en grève. Ilseelles réclament une revalorisation de leurs salaires et l'amélioration de leurs conditions de travail. Pendant 17 jours, dès $4 \mathrm{~h}$ du matin, les salariées se réunissent pour tenir le piquet de grève et organiser la mobilisation. Des soutiens extérieurs à l'entreprise sont également présents en permanence, jouant parfois un rôle de bouclier face aux tentatives de l'employeur de mettre un terme à cette grève, qui connaît un fort retentissement médiatique. Ainsi des Gilets jaunes et d'autres syndiquées des organisations de l'Union syndicale Solidaires (USS) accompagnent quotidiennement les éboueurseuses aux portes de l'entreprise.

2 Dès le premier jour, les éboueurs-euses syndiquées à l'Union syndicale Solidaires et des soutiens capturent des images (photographies et vidéos) de cette mobilisation. Les 14 jours de la grève seront donc particulièrement bien documentés, permettant des publications quotidiennes d'images sur les réseaux sociaux de l'USS, images parfois reprises par la presse locale. Une doctorante filmera ainsi presque quotidiennement le piquet et la lutte dans une double perspective de recherche et d'automédia pour le syndicat. Considérée par les militantes comme exemplaire, cette grève va être matérialisée par la suite dans un documentaire - à l'aide d'une réalisatrice professionnelle - qui deviendra un moyen de rendre visible à la fois les possibilités de syndicalisation dans le secteur du ramassage des déchets et un syndicalisme de lutte et d'institution porté par l'USS.

3 Nous chercherons ici à expliciter et analyser l'ensemble du processus de rechercheaction-création auquel nous avons participé ${ }^{1}$. Il s'agira ainsi de comprendre comment se construit l'image selon la temporalité de sa médiatisation (pendant la grève et après 
la grève), ses usages et ses lieux de diffusion. C'est ainsi la pluralité d'appropriations et usages de l'image qui constitue le point de départ de ce travail.

D'abord le syndicat utilise les images récoltées pendant la grève comme contrepoint médiatique pour contraindre l'employeur et peser dans les négociations (Mercier, 1994). En effet, par la production d'images propres, l'Union syndicale Solidaires (USS) cherche à orienter le traitement médiatique de la grève (Neveu, 1999), qui - loin d'être exhaustif (Giraud, 2009) - oriente la représentation de l'événement (Champagne, 1984). Leur production s'inscrit dans une logique d'adhésion à la grève des habitantes de la Métropole - usageres quotidien nes de la collecte des déchets - alors même que les images hégémoniques produites par les médias se révèlent particulièrement négatives. Cette remarquable médiatisation s'explique en partie par l'usage généralisé de ce service public dont l'absence est immédiatement ressentie par l'ensemble des foyers métropolitains.

Au-delà de l'usage médiatique, les images vont devenir un enjeu majeur lors de la judiciarisation du conflit. Alors que les salariées entament leur $15^{\mathrm{e}}$ jour de grève, l'employeur saisit le tribunal afin de faire cesser le piquet de grève devant l'entreprise. Les deux parties vont recourir à l'image dans les dossiers juridiques afin d'appuyer leur propos. Les images deviennent ainsi un enjeu de légitimation du mouvement de grève et du rapport de forces dans diverses arènes.

De manière complémentaire aux rôles médiatique et juridique, les salariées vont progressivement -- sans être spécifiquement dirigées en ce sens - s'approprier la caméra et mener ce qui pourrait être qualifié de prémices d'enquête ouvrière. Dès lors, la caméra se transforme pendant la grève en outil de libération de la parole sur les conditions de travail, parole considérée par des syndicalistes comme nécessaire pour la mise à l'action (Bué, Delmas et Merlin, 2014). Ainsi, et au-delà de l'événement, cet usage de l'image permet de dévoiler les expériences individuelles et collectives des conditions de travail. Dans l'intention de faire émerger ce récit, un processus de mise en confiance et de légitimation des connaissances du travail est nécessaire. Un échange s'ouvre avec l'enquêtrice qui doit démontrer l'intérêt de raconter face à la caméra le quotidien, les problématiques rencontrées et la connaissance précise des gestes du travail ; mais aussi faire dépasser les timidités. Progressivement intéressées par ce dispositif, les salariées vont se saisir de la caméra et du microphone pour interroger eux-mêmes leurs collègues. Les salariées deviennent enquêteurs de leurs propres conditions de travail. La manière de questionner va alors se transformer: la connaissance plus fine des situations et des relations avec l'employeur permet d'incarner les maux du travail non seulement à travers celui qui raconte, mais aussi par celui qui interroge.

7 À la demande des militants de l'USS, ces images vont être mises au point par une réalisatrice professionnelle dans l'objectif de construire un documentaire militant permettant de transmettre - notamment par le biais des formations syndicales - une mémoire valorisée de cette lutte. Le processus créatif et la restitution de ce documentaire s'inscrivent dans une logique de cinéma d'intervention sociale, ayant pour objectif non seulement de décrire une réalité sociale, mais surtout d'influer sur celle-ci. La mise en image sert non seulement de levier immédiat à la lutte par sa diffusion médiatique, mais fait également mémoire dans l'organisation et s'érige en exemple de lutte à reconstruire. C'est un rôle idéologique et de développement syndical qui est attribué à cette production cinématographique. 
8 Cette ambition d'intervention sociale peut être mise en parallèle avec de nombreuses grèves qui se voient mises en pellicule à la fin des années 1960 : la grève à la Rhodiaceta de Besançon avec À bientôt, j'espère de Chris Marker et Mario Marret ou encore Classe de lutte tourné par les ouvriers eux-mêmes; les ouvriers des $\mathrm{ARCT}^{2}$ de Roanne qui tournent Oui mai... en 1968 ou encore Dominique Dubosc et Daniel Anselme qui font un vaste travail de réalisation avec les ouvriers de la Pennaroya en 1972. Dans la même veine, Joris Ivens se saisit de la caméra pour raconter les luttes des mineurs, des combattants républicains espagnols ou encore des indépendantistes indonésiens.

9 Au cours de la même période, des syndicats et les comités d'entreprise se dotent d'outils vidéo ou de caméras Super 8 pour documenter leurs luttes et des ciné-clubs voient le jour. Cette appropriation des outils audiovisuels passe également par la formation - faite par des réalisateur.trices - des travailleurseuses à leur usage au cours de grandes grèves. Cette pratique s'inscrit dans le champ des grandes expérimentations cinématographiques des années 1970, comme celle du groupe Medvekine (Muel, 2000). Des expériences similaires voient également le jour en Amérique latine: le groupe Ukamau, Cine Liberación, Cine de la Base ou encore les productions de Migel Littin visent - à la fin des années 1970 - à reconstruire des récits historiques décoloniaux et en opposition aux récits hégémoniques. Parmi les réalisateurs contemporains, on peut également citer Avi Mograbi dont la démarche explicitement militante de dénonciation du racisme et du colonialisme s'appuie sur des prises de vue numériques en caméra légère. Ces cinéastes s'inscrivent bien souvent dans la veine du cinéma direct, mobilisant des dispositifs filmiques légers, en prise directe avec le réel. L'ambition de cette forme documentaire réside dans l'immédiateté et l'absence de "filtre" dans le couple filmeur/filmé (Graff, 2011).

10 À l'instar de ces différentes traditions cinématographiques, l'USS entend produire son propre discours sur la grève, à travers la parole des travailleurs'euses. La vidéo apparaît ici comme l'un des nombreux outils déployés par le syndicat. Elle va permettre de populariser la grève, y compris plusieurs mois, années après. Le contrôle des images est ici un enjeu fondamental dans une perspective de propagande : il s'agit d'un cinéma de contre-information (Bovier F. et Fluckiger C., 2016) qui vise à faire entendre la voix des grévistes au-delà des médias dominants. Ces rapides exemples d'expériences cinématographiques militantes permettent de mettre en perspective l'expérience documentaire de la grève des éboueurseuses dont traite cet article. En effet, les images produites revêtent cette dimension documentaire que l'USS va décider d'exploiter pour la réalisation d'un film. L'objectif syndical est pluriel : créer un outil de propagande (Perron, 1995), mais aussi de formation et de transmission (Mariette, 2011). L'organisation entend ainsi montrer un syndicalisme démocratique qui fonde les négociations et la participation institutionnelle sur la lutte de terrain, tout en mettant en avant l'importance de la parole des salariées sur leur travail. Ce processus, inscrit dans le temps long, va nécessiter de nouvelles prises de vue, mais aussi la construction d'une dramaturgie propre au cinéma documentaire a posteriori. Cette nouvelle étape interroge les tensions qui peuvent alors exister entre l'exigence de la production filmique et celle de la lutte syndicale.

11 À travers l'étude des différentes étapes de la production des images au service de la mobilisation et de l'USS, cet article vise à analyser les usages pluriels et contrôlés de l'image par les acteurstrices ayant participé à leur production. Dans un premier temps, il sera question de présenter comment les acteurstrices mobilisées utilisent l'image 
comme moyen pour légitimer leurs demandes et renforcer le rapport de force. La démonstration permettra de mettre en évidence le contraste entre le traitement médiatique hégémonique et celui construit par les militantes. Cette première partie sera également l'occasion d'illustrer le déplacement des images dans l'arène judiciaire. Dans un deuxième temps, ce texte vise à montrer la manière dont émerge et se légitime la parole des éboueurseuses sur leurs conditions de travail et leurs revendications. L'étude de l'enregistrement visuel de ces récits permettra de montrer les tensions entre les objectifs de propagande souhaités par l'organisation et les exigences de la création cinématographique.

\section{L'image au service de la légitimation de la grève et du rapport de force}

Dans un premier temps, nous souhaitons rendre compte du traitement iconographique de la grève des éboueurs'euses qui s'est déroulée entre mars et avril 2019. Tout d'abord par l'analyse des images produites par la presse locale pendant le conflit, pour ensuite rendre compte de celles produites par les militantes de l'organisation syndicale qui souhaitent contrôler la représentation de la grève.

\subsection{La construction médiatique de la grève des éboueurs-euses}

\subsubsection{Où sont les éboueurs·euses?}

13 Souvent cantonnées à l'invisibilité, les éboueurseuses vont être mis en lumière par la grève. Paradoxalement, c'est leur absence qui les rend visibles. Cette mise en évidence passe en grande partie par le travail des journalistes qui racontent et représentent en images la grève. Loin d'être neutres, les photos accompagnant les articles mettent en avant notamment les conséquences négatives de la grève pour les habitant.es. Ces représentations opèrent un glissement du sujet de la grève vers ses conséquences, quel que soit le contenu de l'article. Les journalistes déplacent ainsi le problème par l'image en faisant apparaître comme sujet principal les poubelles qui débordent. Le travail des éboueurseuses, les conséquences du métier sur les corps, les raisons de la mobilisation, les revendications ou les piquets de grève sont pratiquement absents des illustrations. Ainsi, malgré le suivi médiatique local de cette grève, les éboueurs'euses sont pourtant rendues invisibles (Corteel et Le Lay, 2011) par les médias lors des moments de conflit avec l'employeur pour l'amélioration des conditions de travail et l'augmentation de leurs salaires. L'analyse des illustrations de 115 articles de la presse locale - publiés entre le 27 mars 2019 et le 19 avril 2019 - met en évidence cette construction médiatique de la grève. En effet, $60 \%$ des articles sont illustrés par une photo des bacs qui débordent de déchets dans les rues de la métropole. Seulement $10 \%$ des articles représentent des salariées sur le piquet de grève et $6 \%$ montrent les éboueurseuses au travail. Les pourcentages sont encore moindres quand il s'agit de rendre visibles les manifestations dans la ville où se trouve un nombre important de salariées et de soutiens. On note par ailleurs que nombre de ces illustrations ne sont pas issues du mouvement en cours, voire ont été prises dans d'autres villes où les grèves d'éboueurseuses sont plus fréquentes. Les mêmes photographies (pour 
beaucoup issues des mêmes banques d'images) sont utilisées pour illustrer une multitude d'articles de divers médias et à différentes dates.

Graphique 1 : Représentation journalistique de la grève des éboueurs·euses de mars-avril 2019

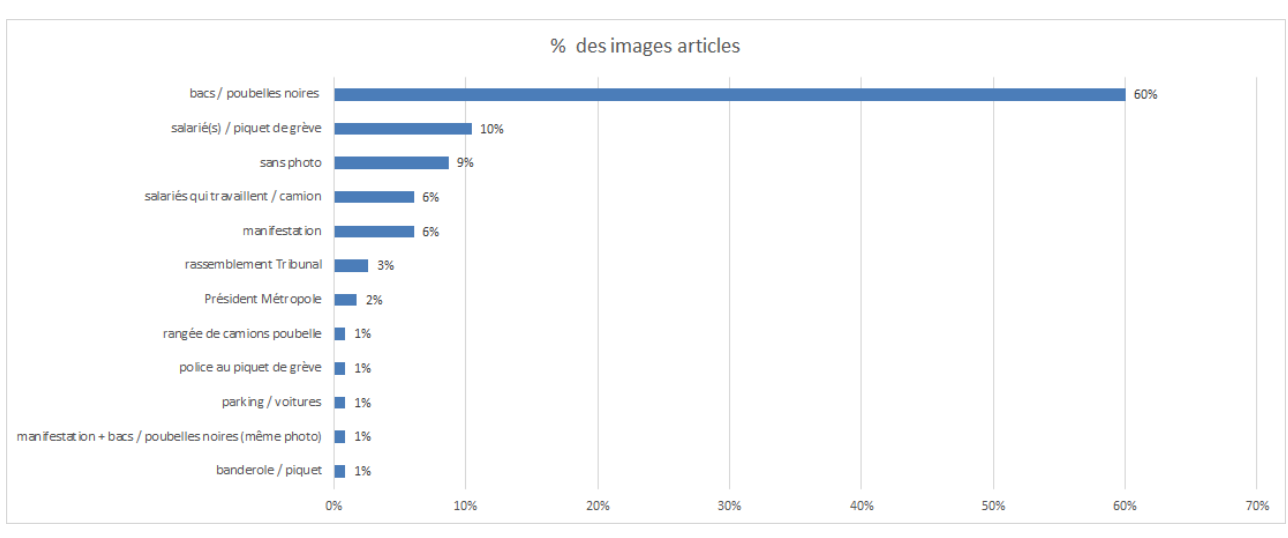

Source propre à partir de l'étude des articles.

Si les médias peuvent être un outil indispensable pour la mise en lumière du conflit et déterminer la réussite de la contestation (Mercier, 1994), ils contribuent parfois à construire une image négative du mouvement et tendent à occulter les moments ordinaires de la mobilisation. Restreindre la représentation de la grève aux poubelles qui débordent contribue non seulement à occulter le travail des salariées mobilisées, mais aussi la pluralité des modalités d'action : aborder les passantes, distribution de tracts, conférences de presse, rassemblements, interpellation des pouvoirs publics, piquets de grève... Cette représentation répond en réalité à une exigence "dramatique" de la presse : c'est le caractère sensationnel de l'information qui est d'abord recherché. Cette recherche du spectaculaire peut s'analyser à l'aune des contraintes structurantes du travail journalistique. Dans cette perspective, le questionnement ne porte pas sur la représentation "adéquate" ou "déformée" de la réalité, mais surtout sur les logiques propres au champ journalistique qui contribuent à définir la manière dont l'information va être traitée et médiatisée (Neveu, 2010). De même l'institutionnalisation d'une "valorisation des images choc, d'une dimension émotionnelle » (Neveu, 2010) permet de pointer les logiques internes de définition de la "bonne information" à publier. Les transformations dans la formation et le profil des journalistes sont également des variables explicatives du traitement médiatique de la conflictualité sociale :

Jadis lié aux mondes syndicaux par sa trajectoire biographique, plus d'une fois
autodidacte, le journaliste social avait pour objet privilégié les conflits du travail, le
syndicalisme, l'industrie. Aujourd'hui diplômé de l'université, le journaliste social
associe plus à cet adjectif les mécanismes du welfare state, une dimension
économique. Son carnet d'adresses est plus riche de portables dans les cabinets
ministériels, les universités, les cadres de ministères sociaux que chez les
syndicalistes ou activistes. (Neveu, 2010)

Ainsi la manière dont les journalistes se forment, leurs réseaux de contacts pour alimenter les informations, mais aussi les logiques hiérarchiques internes et l'institutionnalisation des "bonnes informations" à publier, sont des éléments importants à prendre en compte pour l'analyse. Si cet article ne propose pas d'enquêter sur les logiques particulières au champ journalistique du traitement médiatique de la grève des éboueurseuses à Lyon en 2019, mobiliser les travaux de la sociologie des 
mouvements sociaux sur le sujet permet de poser des jalons d'interprétation sur l'invisibilité des salariées, de leurs conditions de travail, de leurs revendications et des moments routiniers de la mobilisation dans les journaux ayant traité le sujet. Mentionner les interactions entre les différents acteurs (journalistes, politiques, patronat, travailleurs.euses...) permet également de rendre visible la manière dont les grèves - notamment dans la fonction publique - sont généralement traitées. Ainsi, pour les médias, les cheminots "prennent en otage" les usageres du rail ; les agents des collectivités locales "perturbent" le fonctionnement des cantines pour les écolier-es ou encore les organisations syndicales mènent des "guérillas sociales".

16 Cette orientation médiatique péjorative axée sur les nuisances est un véritable marronnier, particulièrement de la presse locale quand il est question de mobilisation dans les services publics de manière générale (transports, éducation, hôpital...).

\subsubsection{Rendre visibles les travailleurs·euses et les raisons de la colère}

17 La formule "d'associés rivaux" (Champagne, 1999) illustre de manière pertinente la relation parfois ambivalente entre les grévistes et les journalistes, imbriquant coopération et concurrence. Le rapprochement de ces deux termes a priori opposés permet d'analyser les tensions de la relation entre les éboueurs'euses et les journalistes. Les images de la grève mobilisées par la presse mettent en évidence un décalage entre l'information publiée pour les journalistes (les poubelles dans la ville) et celle mise en images par les salariées mobilisées (piquets de grève, revendications...). Alors, pourquoi faire appel à des journalistes et souhaiter la publicisation de la grève alors que l'image retenue est surtout celle des nuisances? Pour répondre à cette question, cet oxymore d'associés rivaux à toute sa place. Aux rivalités dans la manière de traiter l'événement s'imbrique le nécessaire recours aux médias pour revendiquer les actions auprès d'un public élargi, comme moyen de pression auprès de l'employeur et de la métropole de Lyon, tout en contribuant à faire durer la mobilisation dans le temps. En effet, ces conflits localisés ne se construisent pas uniquement par les actions collectives des salariées (Béroud, Giraud et Yon, 2018), mais aussi par leur diffusion.

Cette relation ambiguë est traversée par une volonté de contrôler la mise en récit de la mobilisation. Bien que les journaux locaux s'avèrent ici nécessaires et souhaités par les grévistes, les militantes de l'USS - pour certain'es animées par une méfiance vis-àvis de la production du discours et des images par les médias dominants - vont également construire et diffuser une image propre de la mobilisation à travers les réseaux sociaux. Image dont le rôle sera d'expliciter les motifs de la grève au-delà de l'événement. Malgré le manque de formation à l'usage des réseaux sociaux et leur maitrise inégale par les syndicalistes (Olivesi, 2013), la grève des éboueurs-euses suivie par l'Union syndicale Solidaires du Rhône va être exemplaire quant à l'investissement de ces réseaux notamment Twitter et Facebook. Cet usage quotidien sera possible grâce à la présence permanente d'un stagiaire en Master 2 de droit qui s'est chargé de recueillir les images et les textes en vue de leur publication sur les réseaux.

On voit ainsi apparaître sur Facebook et sur Twitter des visages (souvent floutés), des camions poubelles à l'arrêt, des rassemblements des salariées devant l'entreprise. Ces images des grévistes, le piquet devant l'entreprise, les moments de démocratie syndicale ou encore les instants de sociabilité routiniers de la grève comme les repas du midi, prennent le dessus sur les photos qui montrent des poubelles entassées. Cette stratégie de présentation de soi (Goffman, 1973) vise à créer une représentation plus 
humaine et collective du conflit et à rallier les habitant-es et usager-es à la cause quand la fabrique journalistique de l'événement rend invisible le rapport au travail qui se joue. Ce contrepoint aux marronniers du photojournalisme met également en lumière les revendications des salariées sur les réseaux sociaux, quasiment absents du corpus constitué et analysé plus haut.

\subsection{Mobiliser et montrer le rapport de force}

\subsubsection{Du piquet de grève à l'espace virtuel}

Les réseaux sociaux vont également être un moyen d'inciter le plus grand nombre à soutenir la mobilisation. Pour ce faire, il apparait nécessaire de légitimer les revendications et montrer que la cause des salariés est "juste». Les sites internet, les listes de diffusion et autres moyens de communication émanant de l'USS et/ou des grévistes, deviennent ainsi une véritable vitrine (Sinigaglia, 2012) du collectif, que les grévistes et les militantes se doivent d'entretenir. Les photos publiées contribuent ainsi à la production propre des événements avec des logiques «qui ne sont pas forcément en phase avec les cadrages informatifs des médias » (Mercier, 2018). On trouve ainsi sur le compte Twitter de l'USS du Rhône des photos et vidéos de leurs actions, des comptes rendus, déclarations, etc. Le syndicat entend ainsi donner une image contrôlée du groupe, fidèle et qui doit être un levier de mobilisation au-delà de l'entreprise. Par exemple, les militantes incitent ainsi les usager-es de Twitter à appeler Pizzorno pour demander des négociations avec les salariées en grève.

Figure 1 : Échange sur le réseau social Twitter entre l'USS et un usager

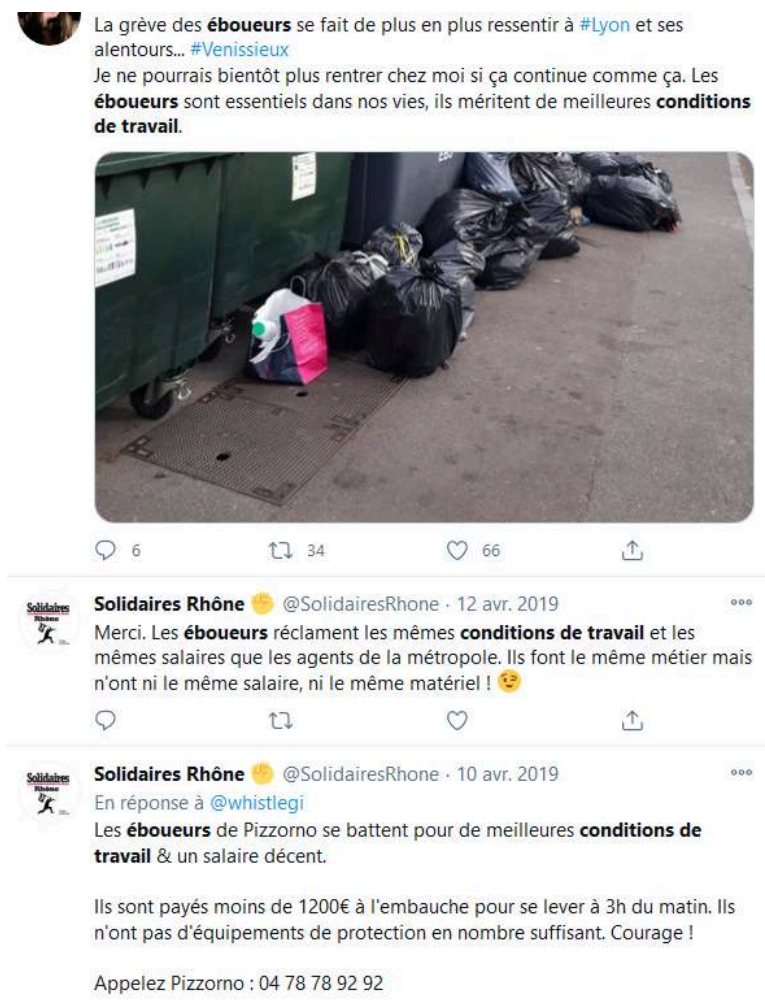

Source : compte Twitter Solidaires Rhône. 
Cette appropriation des outils de communication permet de répondre par l'image à la vision dominante de l'événement construite par les médias locaux. Cette occupation propre de «l'espace virtuel» (Sinigaglia, 2012) transforme les réseaux sociaux en un lieu de mobilisation en soi par l'interpellation directe des habitantes. La présence virtuelle des militants contribue aussi au développement de "l'internet militant» (Granjon, 2001), prouve leur autonomie vis-à-vis des politiques ou des journalistes et contribue à la création et au maintien du rapport de force.

Montrer le nombre de salariées et de personnes en soutien - étudiant-es, Gilets jaunes, camarades du syndicat, etc. - sur le piquet de grève, les feux devant les grilles de l'entreprise, les manifestations dans le centre-ville, les rassemblements devant le siège de la métropole sont autant d'illustrations de la force de la mobilisation et sa radicalité. En effet, les grévistes lancent des appels à la mobilisation en direction des militantes du syndicat Solidaires local ou plus largement aux habitantes métropolitaines afin de faire la démonstration du soutien dont ilselles bénéficient. Ces images vont être diffusées dans les réseaux syndicaux et au-delà; les salariées vont ainsi pouvoir exprimer leur volonté de poursuivre la grève jusqu'à l'ouverture des négociations et la satisfaction de leurs revendications. Le processus de mobilisation passe en grande partie par la production et la diffusion d'images : sur le piquet, mais aussi pour montrer leurs actions vers l'extérieur, au-delà des portes de l'entreprise. Aussi, le soutien d'un nombre important d'usageres, de camarades, va conforter les grévistes dans leur légitimité et permettre de consolider le mouvement et le rapport de force avec l'employeur.

Figure 2 : Manifestation du 15 avril en soutien à la grève : cortège de l'USS

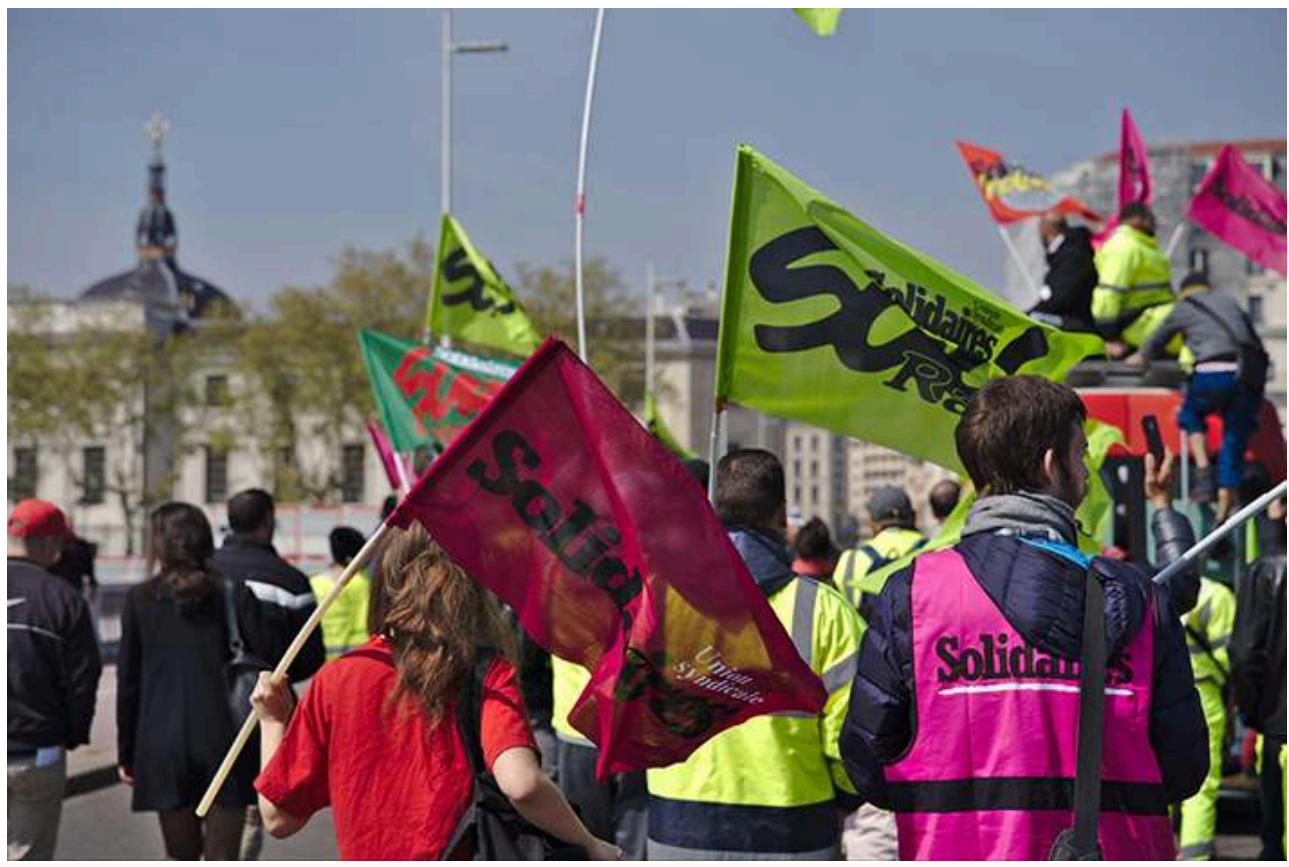

(c) Yanis Cernesse. 
Figure 3 : Des militant·es de l'USS sur le piquet de grève

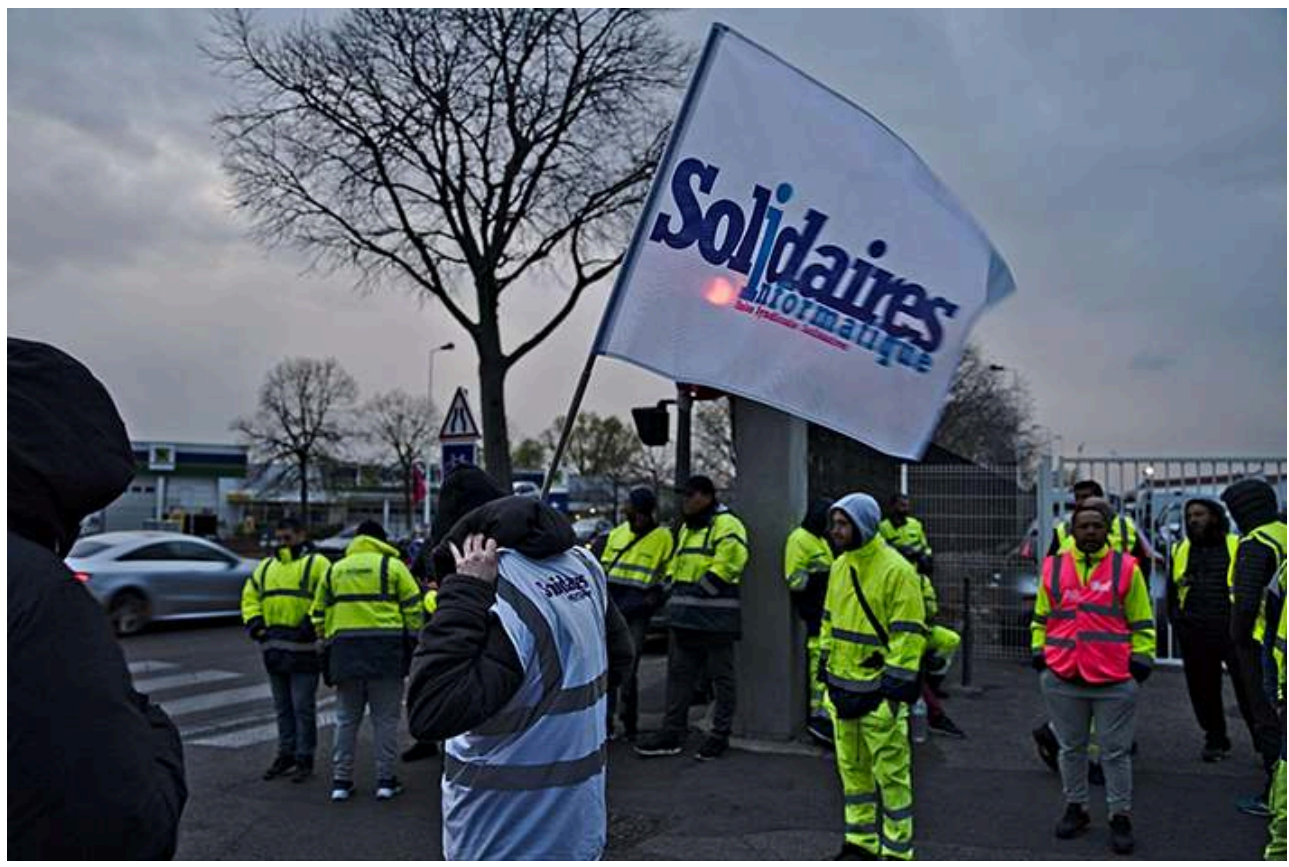

(c) Yanis Cernesse

\subsubsection{Usages de l'image dans l'arène judiciaire}

Alors que les salariées entament leur $15^{\mathrm{e}}$ jour de grève, Pizzorno saisit la justice par l'assignation en référé d'heure à heure aux fins « d'expulsion des salarié'es grévistes du site $^{3}$ " de dix salariées - dont deux sont des représentants du personnel - pour " entrave à la liberté de travail, menaces et intimidations, violences, sabotage et incendie volontaire ». Cette "judiciarisation " (Pelisse, 2009) du conflit va également être l'occasion pour l'une et l'autre des parties de mobiliser les images produites durant la grève. Nous avons vu l'importance de ces représentations sur le plan médiatique et pour construire le rapport de force : elles deviennent désormais un enjeu essentiel de la bataille judiciaire. L'image se déploie sur tous les fronts. Pour les salariées et les militantes solidaires, cette action en justice va à l'encontre du droit de grève :

L'entreprise a choisi au hasard des salariés pour essayer de faire pression sur la grève, et de s'attaquer aux droits de grève. Elle veut obtenir que les grévistes ne puissent plus se rassembler avec leur piquet de grève, prétendant que c'est un obstacle à la liberté du travail.

Le responsable de l'union syndicale déplore que l'entreprise fasse appel à des intérimaires et réclame l'ouverture de négociations :

Depuis 16 jours, il y a zéro négociation. L'entreprise se contente d'appeler la police, des huissiers, des avocats et essaie de criminaliser un mouvement de grève, alors que la grève est légale en France.

(Article Rue89, entretien avec Frédéric Leschiera, responsable de l'USS du Rhône, 19 avril 2019)

En entrant dans l'arène judiciaire, le confit suscite encore la mobilisation de photos dans les dossiers des deux parties. Du côté de l'employeur, l'avocat s'appuie sur les photos prises par les huissiers, pour accompagner les procès-verbaux et ainsi tenter de prouver les accusations à l'encontre des salariées. 
Vers $6 \mathrm{~h} 40$, les grévistes pour la plupart en gilet jaune et un orange bloquent un véhicule conduit par Monsieur Polin transportant des personnes souhaitant travailler qui devaient être amenées sur leur lieu de travail (cf. photo 2). [figure 4]

Figure 4 : « Blocage de l'accès au site » (constat du 3 avril 2019)

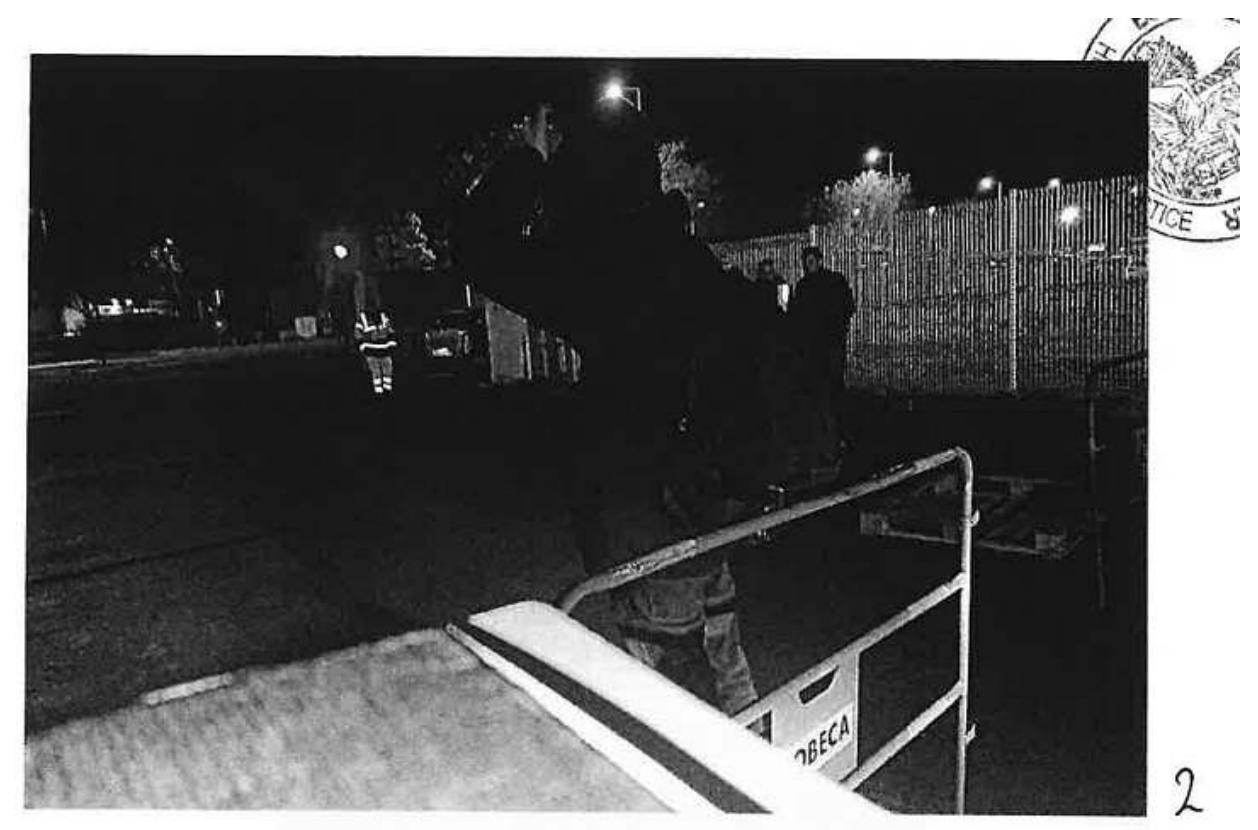

Source : procès-verbal versé au dossier par le plaignant (constat d'huissier du 3 avril 2019).

Sur cette photographie en blanc et noir produite au dossier, on aperçoit des barrières et quelques personnes devant des grilles. Mais les huissieres vont aussi apporter des images du feu ou encore des poubelles renversées devant le portail pour appuyer leur démonstration à l'encontre des salariées. Cette construction de l'événement - par la mobilisation des moments extraordinaires de la grève - pour appuyer leur argumentaire devant la justice poursuit l'objectif de faire condamner les salariées et mettre ainsi un terme au piquet de grève devant la société. Car c'est bien là l'enjeu de cette assignation pour Pizzorno : faire cesser la démonstration visible de la grève en empêchant la tenue du piquet. Les revendications des grévistes doivent être rendues invisibles pour le public et les usageres de la collecte de Pizzorno; en ce sens, la présence devant les grilles de l'entreprise nuit à l'image du groupe. Pizzorno enlèvera d'ailleurs l'enseigne de l'entreprise sur ses grilles très rapidement après le début du conflit afin d'éviter que le nom du groupe soit visible sur les images de la grève. Dans la même perspective de contrôle de l'image du groupe, le protocole de fin de conflit stipule que les «organisations syndicales retireront les banderoles et panneaux apposés sur la grille d'entrée de l'Agence ». 
Figure 5 : Foyer devant le piquet de grève

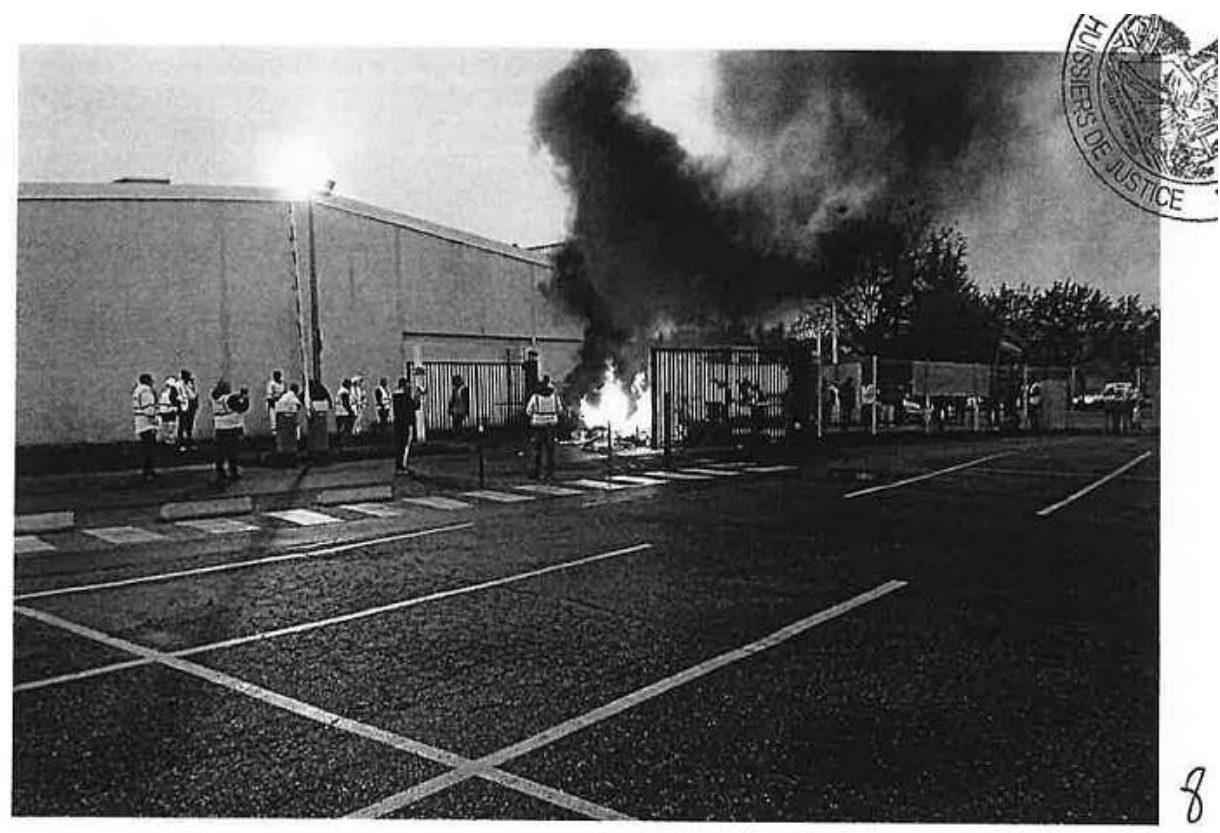

Source : procès-verbal versé au dossier par le plaignant (constat d'huissier du 3 avril 2019).

L'avocate qui défend les salariées va également se munir d'images produites par l'organisation syndicale ou la presse locale afin d'appuyer son argumentaire. Des captures d'écran du compte Twitter de l'organisation syndicale montrent que le portail de l'entreprise reste ouvert malgré le piquet par exemple. Ici, l'enjeu est de convaincre la cour de la légalité de la grève et ainsi renverser l'argumentaire de la partie adverse. Cette opposition des images a pour but de légitimer d'un côté et de l'autre les accusations et la défense. 


\section{Solidaines Solidaires Rhône \\ $\widehat{h}$... @SolidairesRhone}

\section{C'est enfin ouvert après plusieurs échanges entre la police, la sécurité et la direction. \#Pizzorno \#Greve \#Eboueurs \#Lyon}

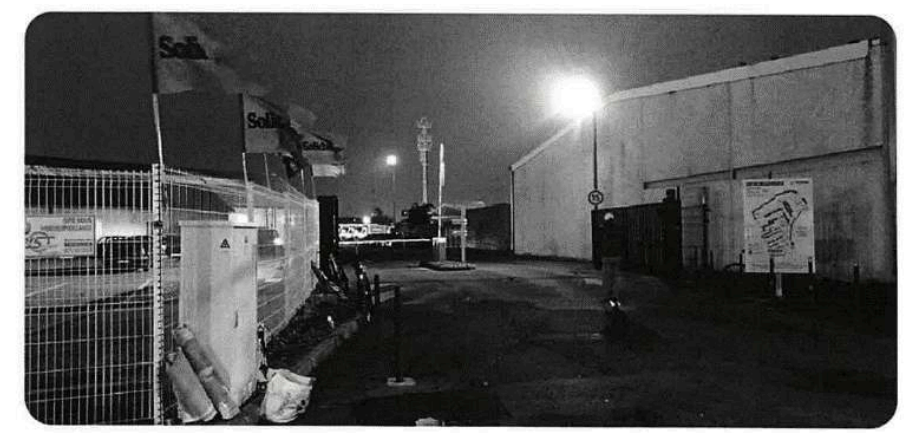

\section{4:58 $\cdot 11$ avr. 19 $\cdot$ Twitter for Android}

Source : compte Twitter Solidaires Rhône.

Cet affrontement judiciaire se conclut par la victoire des salariées. La justice considère que l'entrave à la liberté du travail n'est pas caractérisée puisque les camions sont en mesure de sortir de l'entreprise même si c'est avec quelques heures de retard.

Cette première partie a permis de montrer et comprendre l'utilisation, pendant le conflit, des images produites par les médias et par l'organisation syndicale. Si la presse locale écrite construit de par ses illustrations iconographiques une représentation négative de la grève, l'USS de son côté investit quotidiennement les réseaux sociaux afin de contrebalancer cette première vision. En agissant sur la scène médiatique et judiciaire pendant la grève, l'USS entend renforcer le rapport de force face à l'employeur.

\section{La parole des éboueurs-euses comme socle de l'action syndicale}

Le regard portera ici sur des usages proprement internes à l'organisation dans le but de promouvoir un type de syndicalisme qui légitime la parole des salariées. La caméra devient un révélateur de la parole et permet aux salariées de s'exprimer sur leurs conditions de travail et même de s'approprier l'outil pour raconter leur quotidien du travail. 


\subsection{La caméra comme révélateur du travail quotidien}

\subsubsection{Faire confiance et se faire confiance : les conditions d'émergence d'une parole sur le travail}

31 Au fur et à mesure du tournage, et comme c'est souvent le cas en documentaire, notre caméra a, pour ainsi dire, trouvé sa place. Elle n'a pas disparu, n'est pas devenue invisible, mais s'est transformée en protagoniste à part entière de la grève. Protagoniste, en tant qu'elle permet, immédiatement - comme nous venons de le démontrer - de produire des images propres de propagande. Elle joue un rôle pour la grève. Elle s'est donc déjà révélée une alliée objective des grévistes en devenant un outil pour le développement et la communication autour et pour leur lutte. Si nous utilisons ici une métonymie, c'est évidemment le couple filmeur/caméra dont il est question. La relation de confiance qui se joue entre les protagonistes et la personne qui manie la caméra est déterminante dans le processus de création filmique, elle est constitutive du genre documentaire. C'est le regard du réalisateur qui se révèle à travers les images filmées. Sa relation avec les grévistes, le respect des choix des protagonistes quant à l'exploitation de leur propre image comme la présence dans la durée sont autant d'éléments fondamentaux pour la réussite du projet documentaire. Ils déterminent la confiance et l'appropriation de l'outil par les premieres concernées. Une fois dépassée la légitime défiance qu'elle produit - la caméra est d'abord un dispositif des médias dominants qui posent rarement un regard bienveillant sur les mouvements de grève - la caméra devient donc participante (Heusch, 1992). Elle est participante en ce qu'elle est aussi portée par une camarade du syndicat. À l'instar de René Vautier qui interpelle directement un commissaire dans Quand tu disais Valéry (1975), la cadreuse s'adresse directement à l'huissière de justice à plusieurs reprises. Cette implication s'avère parfois indispensable pour construire la relation de confiance, le contrat implicite ou explicite entre filmeur et filmé.

Figure 7 : Capture d'écran vidéo d'une confrontation avec l'huissière autour de la place de la caméra

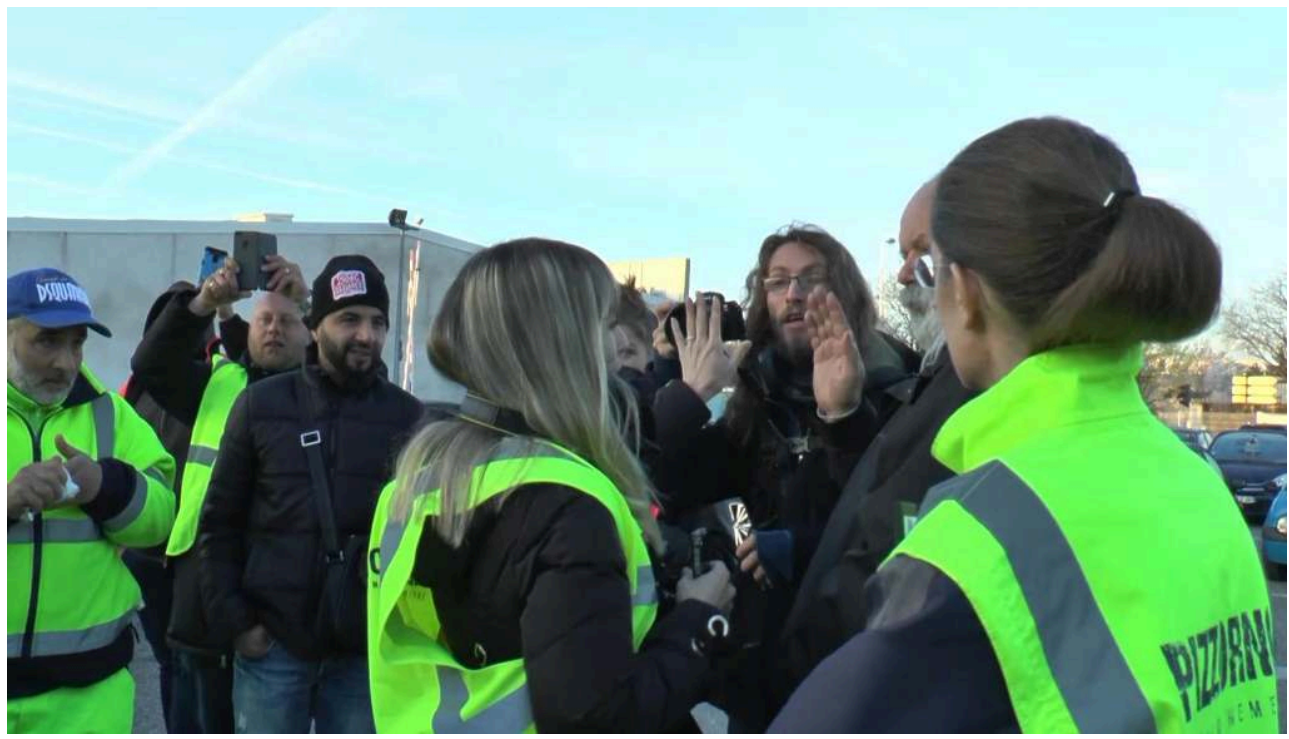

Source : extrait vidéo des rushes de l'USS [https://vimeo.com/662014345/32f0043cfd]. 
fois cette place bien définie, cette confiance établie, la caméra peut enfin jouer son rôle de révélateur. Le dispositif intimidant, parfois même hostile, devient alors un outil au service de la lutte. La présence de la caméra ouvre de nouvelles perspectives : elle place au premier plan la parole des grévistes, leur offre le moyen de s'exprimer dans un cadre souple, et permet ainsi de capter des séquences qui n'auraient peut-être pas émergé en l'absence de dispositif filmique. La réticence du départ - notamment à considérer sa propre expérience comme significative, intéressante pour le collectif - est peu à peu remplacée par un désir d'expression.

ne considèrent pas d'abord par eux-mêmes comme détenteurs d'une parole légitime à partager. Ils répondent le plus souvent qu'ils n'ont "pas grand-chose à dire". La documentariste va devoir lever le voile et permettre d'opérer un basculement vers une appropriation des protagonistes de la médiatisation de leur propre vécu. Pour la grève des éboueurseuses de Pizzorno, cette appropriation sera particulièrement visible : les grévistes eux-mêmes finiront par solliciter la présence de la caméra pour montrer le quotidien sur le piquet ou tout simplement témoigner. Ce basculement va permettre de mettre en lumière, tout particulièrement, les conditions de travail des grévistes. Ceux-ci et celles-ci vont alors, assez spontanément, décrire leur quotidien face à la caméra, ou encore montrer, sur leurs corps, les stigmates du travail. La grève marque une première rupture avec le quotidien, elle constitue un événement. La présence de la caméra vient alors appuyer cette rupture, elle permet de saisir l'importance du moment en offrant un cadre aux grévistes pour penser leurs propres conditions matérielles d'existence comme un sujet en soi. La caméra permet d'opérer un déplacement du regard sur son propre quotidien.

De la même manière, la conférence de presse du 5 avril 2019 à la Bourse du travail, en mettant la parole des grévistes au premier plan, va agir comme un révélateur. La mise en scène, la présence de journalistes, va conforter les grévistes dans leur légitimité : le récit de leur quotidien apparaît comme constitutif d'un récit collectif, récit du travail puis de la lutte. Les protagonistes témoignent parce que des caméras sont présentes: l'image est l'un des supports essentiels de la revendication.

\subsubsection{Raconter par l'image pour revendiquer}

Images du travail, travail des images, 12 | 2022 
Figure 8 : Capture d'écran vidéo d'un gréviste montrant une blessure à l'acide provoquée par les produits de nettoyage

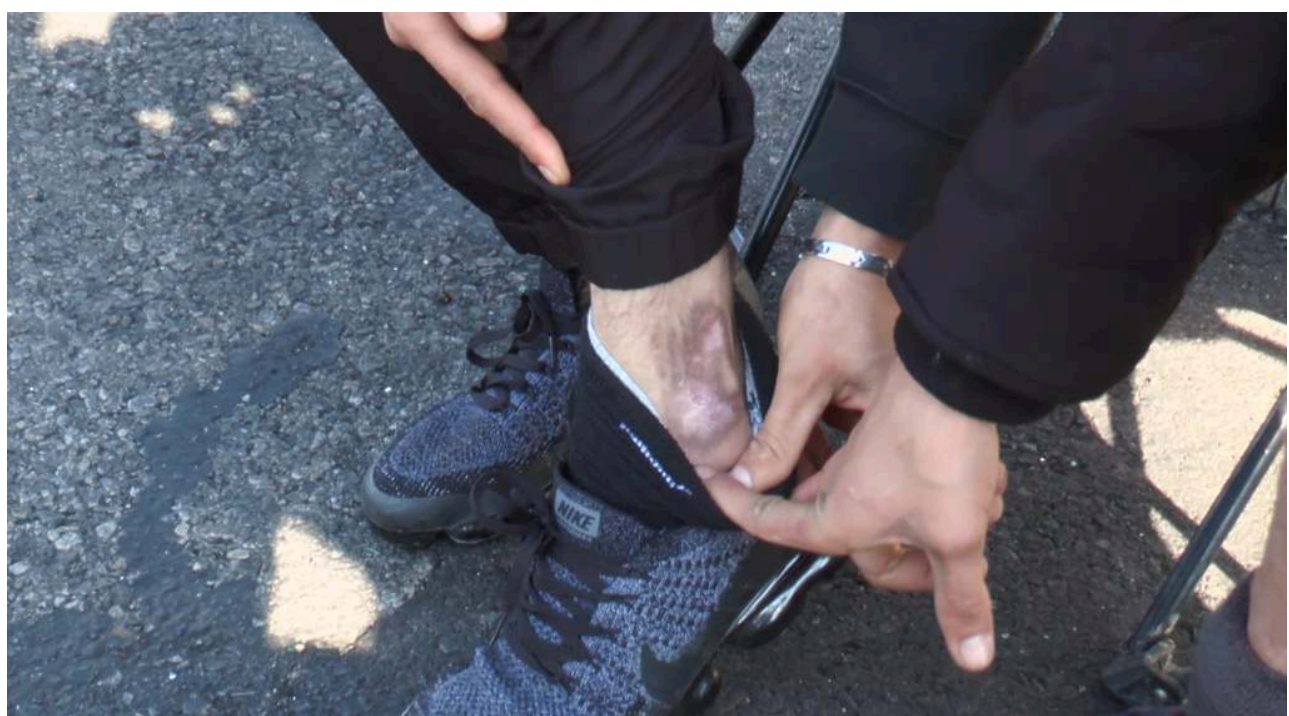

Source : extrait vidéo des rushes de l'USS [https://vimeo.com/662019602/2f6ec988ec]

À mesure que la grève avance, les temps de discussions et d'échanges se font plus nombreux. Les grévistes se connaissent mieux, nouent des amitiés. Le terrain se transforme : le piquet fait désormais partie du quotidien. L'événement n'étant plus le piquet en lui-même, la cadreuse-réalisatrice va pouvoir utiliser les nombreux « temps faibles » (Depardon, 1993) du tournage pour approfondir son enquête. Des entretiens en situation vont permettre de décrire les conditions de travail avec une relative précision. Ces nouvelles interviews vont assez rapidement donner à voir l'ancrage des revendications des salariées. Des thématiques récurrentes reviennent, notamment autour des maux physiques liés au travail : une chauffeure raconte ses douleurs de dos, un ripeur explique comment il s'est gravement brûlé avec des produits non dilués en nettoyant la cuve du camion (l'une des revendications des grévistes porte sur ces lavages pratiqués dans des conditions particulièrement dangereuses), etc. Les unes et les autres décrivent les gestes du quotidien, les rapports avec leurs supérieurs, le manque de matériel. Ces témoignages font apparaître la nécessité, lors de la longue phase de dérushage, d'aller filmer les - désormais anciens - grévistes lors de leurs tournées de collecte.

Un des éboueurs filmés a posteriori est Éric. Sorteur à Pizzorno, Éric commence sa tournée à $5 \mathrm{~h}$ du matin. Il fait encore noir et les rues du $3^{\mathrm{e}}$ arrondissement de Lyon sont vides : aucune voiture ou passante ne viennent troubler le calme de l'aube. L'éboueur est chargé de sortir les bacs de poubelle pleins des immeubles et de les poser au bord des trottoirs pour que ses collègues ripeurs puissent les vider dans la benne à ordures $\mathrm{du}$ camion. Pendant que le conducteur et les ripeurs font le tour de l'arrondissement, Éric est de nouveau en train de parcourir à pied les mêmes rues pour rentrer les bacs vides dans les immeubles. Vers $11 \mathrm{~h}$ du matin, tous les bacs vides du $3^{\mathrm{e}}$ arrondissement seront de nouveau dans les immeubles prêts à recevoir de nouveaux déchets. 
Figure 9 : Capture d'écran vidéo d'un sorteur au travail

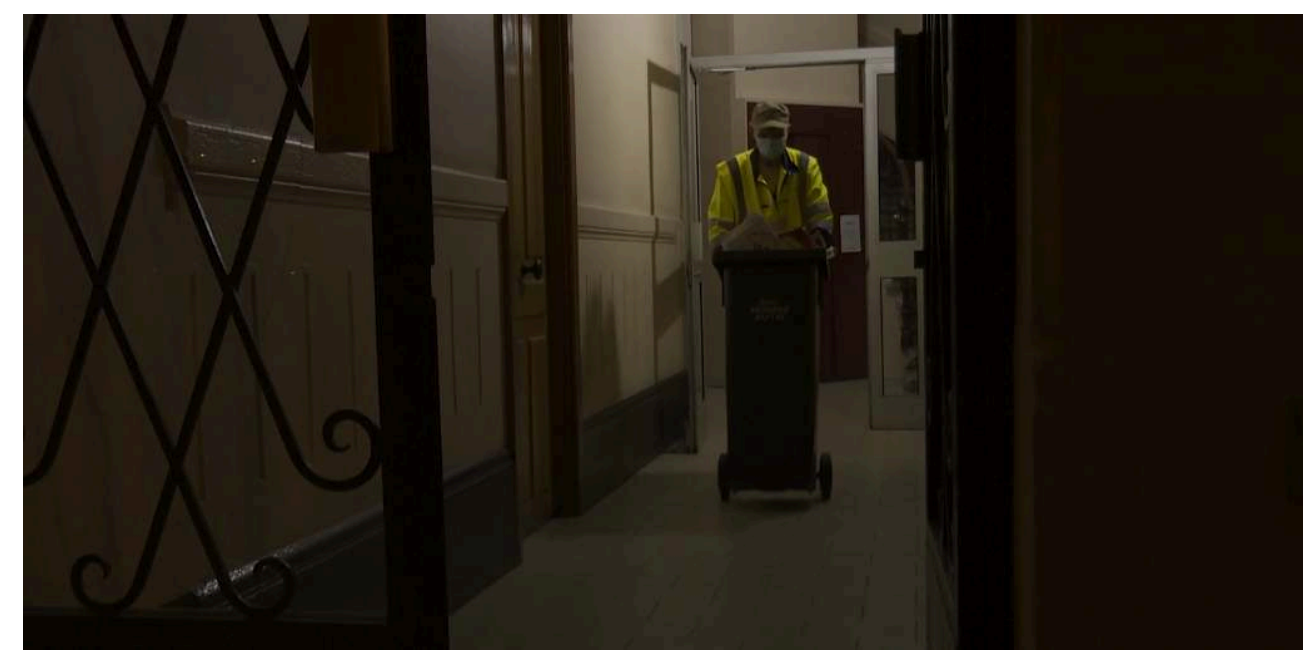

Source : extrait vidéo des rushes de l'USS [https://vimeo.com/662019818/567f0b9c67].

Loin d'illustrer de manière exhaustive le travail des éboueurs-euses, ce passage entend décrire les multiples tâches auxquelles ils s'adonnent dès le petit matin. Cette séquence filmée a été réalisée après la grève afin de rendre visible le travail quotidien des sorteurs-euses. Cette enquête visuelle va permettre de saisir le quotidien, mais aussi et surtout les gestes du travail déjà décrits par les grévistes. Montrer, donner à voir - là où l'invisibilité est souvent de mise - la répétition, la course, les corps en tension et en action, tel est l'objectif de ces images captées hors de la temporalité de la grève.

\subsection{Décrire et comprendre : le documentaire comme support de formation et d'implantation syndicale}

\subsubsection{De la caméra participante au film participatif : prémisses d'une "enquête ouvrière"}

Cette confiance va dans un second temps permettre une appropriation plus grande encore : l'un des grévistes va se saisir de la caméra pour aller lui-même interroger ses camarades. Il s'improvise cameraman mais surtout intervieweur. Une partie des entretiens en situation (sur le piquet) sont le résultat de ce dispositif d'auto-enquête. La caméra jusqu'alors participante devient participative! La plus grande accessibilité des outils de captation vidéo est en ce sens un enjeu fondamental: tout le monde peut désormais produire (et diffuser) son propre contenu. Le dispositif qui se met à l'œuvre dans notre cas n'est pas sans rappeler l'enquête ouvrière si chère à Marx et qui a accompagné le développement des organisations ouvrières depuis le début du XIX ${ }^{e}$ siècle, à Lyon tout particulièrement avec l'Écho de la fabrique dans les années 1830. Ici, les questions sont également posées par les travailleurs euses eux-mêmes -- Marx en introduction à son questionnaire publié en 1880 dans la Revue socialiste nous dit: «[seuls les ouvriers] peuvent décrire en toute connaissance de cause les maux qu'ils endurent » et cela permet parfois d'aller plus loin sur le questionnement qu'avec la cadreuse et/ou la chercheuse. Les réponses entrent donc en résonance les unes avec les autres, permettent d'affiner progressivement cette connaissance du travail et du 
quotidien et favorisent leur expression. Ce dispositif permet également de contribuer à une reconnaissance mutuelle en tant qu'exploitées.

La plus grande accessibilité des outils audiovisuels favorise ces nouvelles pratiques en interne en même temps qu'elle permet une plus grande diffusion vers l'extérieur. Cette révolution numérique est aujourd'hui un élément essentiel dans les nouvelles formes d'organisation de la lutte comme de propagande - des Gilets jaunes au mouvement Black Lives Matter, l'image et les réseaux sociaux occupent une place prépondérante dans le développement de nombre de mouvements sociaux. Ce nouvel usage a d'ailleurs entraîné différentes tentatives pour en limiter la portée du fait de leur potentiel contestataire. L'omniprésence de photographes et vidéastes amateurs ou professionnels dans les luttes est plus particulièrement contestée par les forces de l'ordre. Cette nouvelle revendication des représentantes de policiers a été mise audevant de la scène médiatique durant les mobilisations contre la loi sécurité globale dont l'article 24 entendait pénaliser l'utilisation d'images des forces de l'ordre -- dont le droit à l'image est déjà garanti par l'article 9 du Code civil. Cette tentative de limiter la diffusion, devenue courante, de bavures policières s'inscrit dans une bataille médiatique autour des violences policières. L'image en est devenue l'épicentre, démontrant s'il le fallait son importance dans les conflits en cours.

Nous pouvons ici nous permettre une analogie avec l'arrivée de la vidéo dans les luttes sociales dans les années 1970. Cette innovation technologique favorise l'émergence d'une nouvelle forme de documentation, mais aussi d'autonomie, notamment en permettant de s'affranchir d'un certain nombre de contraintes techniques induites par le cinéma. Elle a été concomitante avec l'émergence de nouvelles formes de luttes et de revendications, contribue à les produire et à les diffuser dans un mouvement réciproque. Le collectif Vidéa ${ }^{4}$ écrit en 1976 :

Châtrée depuis toujours par la société patriarcale, façonnée par le désir de l'homme, "la femme" n'est qu'une image coupée de sa propre identité. C'est par la lutte, par les connaissances acquises sur elles-mêmes, que les femmes sont en train de créer leur propre histoire et leur propre culture [...]. La vidéo est pour nous un moyen de contre-information. Tout ce qui nous concerne doit être dit par nous, et non par les hommes qui, détenant le monopole des médias, dénaturent l'information.

(Vidéa, 1976, p. 147)

Pour René Vautier, c'est de la «mise à disposition d'une classe opprimée des moyens d'expression monopolisés jusqu'ici par la classe dominante » que naîtra "une esthétique neuve" (Vautier, 1977). Il y a donc un véritable enjeu d'autonomie dans l'appropriation du médium. Appropriation qui, pour notre cas, va permettre de construire le récit du travail à partir de la parole des grévistes. Un processus qui n'est pas exempt de tensions.

\subsubsection{Tensions entre les objectifs de propagande et la création cinématographique}

Comme nous l'avons vu, les images de l'USS n'ont pas été tournées avec l'objectif de réaliser un long métrage. Elles ne répondent donc pas, à certains égards, à l'exigence narrative du cinéma documentaire. La documentariste qui interviendra dans un second temps de la réalisation du film (postérieur à la grève) devra donc composer avec ces lacunes dans la construction dramaturgique. Les images sont prises sur le vif, sans écriture préalable. Il faudra donc, au dérushage, s'atteler à l'écriture de cette 
dramaturgie et penser de nouvelles images pour étoffer le récit. L'unité du film se crée tout particulièrement autour du lieu de déploiement de la grève : le piquet. C'est à partir de ce lieu, et à travers une chronologie linéaire, que le documentaire se déroule. L'USS, dans un souci davantage politique que cinématographique, entend proposer ce que l'on pourrait qualifier de « documentaire-exposé5 »en ce qu'il prétend fournir une interprétation complète et d'apparence objective du réel. Il a une visée didactique indéniable (Nichols, 1991 ; Lioult, 2004). Cette forme, relativement prisée en télévision, ne permet pas une grande marge de manœuvre ni sur le plan esthétique ni sur le plan narratif, mais répond précisément aux objectifs de diffusion la plus large possible de la grève, de ses modalités et de ses revendications. En ce sens, il faudra un long travail de discussion et des allers-retours constants durant le montage, entre la documentariste et les représentantes de l'USS. L'exigence informative, parfois très précise de l'USS, doit se conformer aux nécessaires raccourcis, simplifications qu'implique la dramaturgie propre à l'écriture filmique. Le cinéma est aussi un média du dévoilement, de la suggestion. Cette tension entre l'objectif purement politique et l'ambition cinématographique sera au cœur des réflexions lors de cette phase de montage. La nécessité d'utiliser le film comme outil de formation interne au syndicat orientera définitivement les choix narratifs. Ce sont ces objectifs exposés par l'USS qui détermineront les interviews menées après la grève : l'avocate et les délégués seront ainsi sollicitées pour apporter un éclairage juridique pour la première et reconstituer le récit de la grève pour les seconds. Ces deux entretiens structurants permettront d'apporter des explications là où la caméra se cantonnait souvent à un rôle d'observation sur le piquet: outre les entretiens " participatifs » autour des conditions de travail, des interviews en situation auraient pu jouer ce rôle, mais ce besoin n'aura pas été identifié à temps. Cette nécessité d'explicitation des différentes scènes se fera jour quand le syndicat décidera de transformer ces images en documentaire.

\subsubsection{La vidéo en formation syndicale : transmettre l'image d'un syndicalisme de terrain et victorieux}

Pour le syndicat l'enjeu est également interne : il s'agit de former, d'expliquer comment se met en place la grève et ainsi de transmettre les outils aux travailleurseuses.

Ce cinéma d'intervention sociale, qui articule un projet politique, est en phase avec les grèves, les manifestations et les revendications syndicales : énoncé depuis un champ extérieur à la culture cinéphilique, il est diffusé dans des espaces publics indissociables des luttes qui s'y expriment, tels que l'usine, la salle de réunion d'une section syndicale ou encore le lieu d'une manifestation.

(Bovier F. cité par Fleckinger, 2010, p. 47)

De la même manière, l'USS va orienter la diffusion vers des lieux privilégiés de syndicalisation. Le premier public sera donc interne au syndicat. Bien que les projections aient été suspendues en raison de la crise sanitaire, la stratégie de diffusion reste relativement inchangée. Des projections en ligne sont ainsi programmées auprès de différents publics et dans différentes temporalités pour répondre aux différents objectifs de l'USS. D'abord, auprès des grévistes de Pizzorno eux-mêmes, dans une période de fort reflux de la mobilisation dans l'entreprise. Ensuite, auprès de l'ensemble des militantes de l'USS nationalement et enfin une diffusion publique à l'occasion des élections professionnelles des TPE est envisagée. À plus long terme, le film sera utilisé dans le cadre de formations syndicales internes pour expliquer les mécanismes de la grève. Plus généralement, les images ne sont désormais plus 
envisagées comme des accélérateurs de cette grève spécifique, mais bien comme des outils de diffusion et d'élargissement de la lutte, voire d'inspiration pour d'autres grèves.

Enfin, un livret de cinquante-quatre pages a été rédigé après la réalisation du film afin de proposer un support original : le mediabook. Ce livret, édité en couleurs sur papier glacé, apporte à la fois des éléments contextuels et d'analyses complémentaires au film, mais aussi de nombreuses photographies du conflit. Il contient un QR code et une clé USB permettant d'accéder au film. L'usage de ce livret est d'abord interne puisqu'il sera distribué lors de formations syndicales. Une deuxième commande à partir de ces images est ensuite envisagée par l'USS : utiliser les entretiens "participatifs » pour réaliser de courtes vidéos de formation autour de thèmes spécifiques -- EPI, sécurité, droit de grève, etc. Il s'agit donc d'exploiter les images tournées durant la grève au maximum. Le long travail de montage implique de nombreux choix tant dans la construction que dans les coupures. Ici, l'USS récupère une partie du matériau brut pour répondre à d'autres objectifs. Sur la vingtaine d'heures de rushes, plus de dix-neuf ne seront ainsi pas utilisées pour le film (une proportion à peu près toujours identique pour ce genre de documentaire-exposé). Des séquences non utilisées vont pouvoir trouver une seconde vie à travers ces courtes pastilles didactiques.

Figure 10 : Capture d'écran d'une pastille de formation sur les EPI

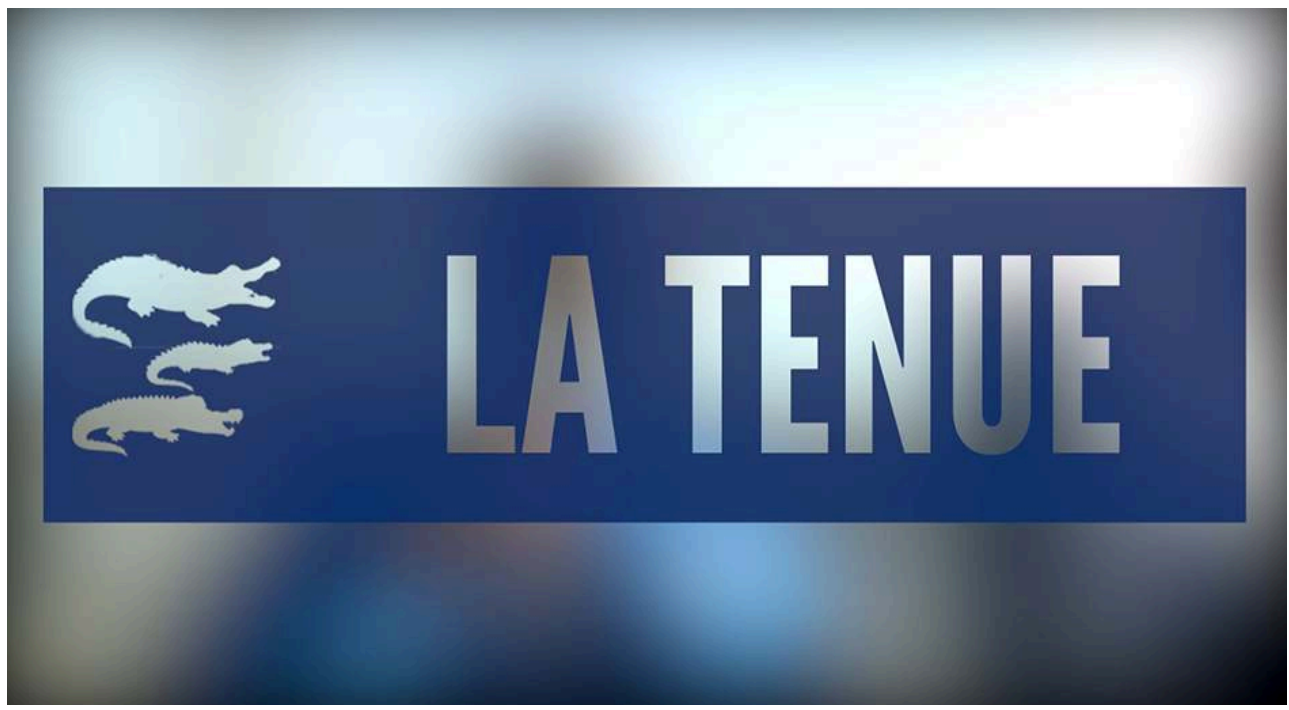

Source : pastille de formation syndicale [https://vimeo.com/662020059/915aa5e265]

\section{Conclusion}

Analyser les productions photographiques et filmiques réalisées pendant et après la grève permet de montrer les différents usages et effets que ces images ont eus sur la mobilisation, mais aussi sur les salariées eux-mêmes. En effet la production d'images photos et vidéos va permettre de construire une représentation propre et contrôlée de la lutte qui entre en dissonance avec l'image hégémonique des médias dominants (une mise en œuvre militante qui mérite d'être mise en avant, notamment dans un contexte de discussion de la proposition de loi sécurité globale, un projet sécuritaire et restrictif dans l'usage des images). Au-delà de cet usage symbolique du conflit, ces supports 
jouent également un rôle important lors de la judiciarisation de la lutte par l'employeur qui décide de réprimer dix salariées pour « entrave à la liberté de travail, menaces et intimidations, violences, sabotage et incendie volontaire». L'avocate des salariées mobilise dans le dossier juridique les photos et les articles de journaux qu'elle trouvera pertinents pour contester les accusations. Ces deux investissements - symbolique et judiciaire - de l'image sont imbriqués avec celui de la fabrication du rapport de force face à l'employeur. Il s'agit en effet de montrer en direct sur les réseaux sociaux qu'ils sont nombreux'ses et qu'ils investissent une diversité de répertoires d'actions : piquets de grève, manifestations, conférences de presse, etc. Cet effet des images dans le rapport de force devient d'autant plus important quand les salariées revendiquent publiquement leur victoire face à l'employeur dans l'arène judiciaire.

En plus de contribuer à légitimer la grève, la caméra sert de support de valorisation de la parole des salariées. Le processus de mise en confiance des éboueurs'euses face à la caméra n'est pas anodin. En effet, la personne qui filme doit transformer un dispositif intimidant qui donne généralement la parole aux dominants, en un moyen de mise en valeur du récit du travail et des raisons de la colère des éboueurseuses. Ainsi il s'agit de faire en sorte que la parole des grévistes, qu'eux-mêmes considèrent à première vue comme illégitime ou insignifiante, soit par la suite enregistrée et valorisée comme un élément essentiel de la lutte. Ici la relation de confiance construite pendant la temporalité de l'action est essentielle. Cette démarche est clairement orientée par la doctorante qui s'intéresse au travail concret des éboueurseuses, mais aussi par certain'es militant-es de l'Union syndicale Solidaires qui souhaitent mettre en avant ce quotidien et détailler des conditions de travail souvent invisibles. C'est donc ce dispositif d'interview sur le moment de la grève élaboré par les soutiens, mais aussi par les salariées eux-mêmes qui permet de qualifier la démarche de prémices d'enquête ouvrière. Une manière de prendre place, qui va permettre non seulement de valoriser les connaissances des travailleurseuses, mais aussi de faire de l'image un outil pour le développement syndical. Ce dernier usage va se matérialiser sous la forme d'un documentaire, de pastilles pour la formation syndicale et d'un mediabook. Ces deux derniers supports vont être réalisés par une documentariste professionnelle qui devra compléter les images et construire une dramaturgie propre à la narration et à l'esthétique du documentaire. Ce processus nécessite plusieurs allers-retours entre les militantes de l'organisation et la réalisatrice qui devra composer avec les attentes de l'USS et ses propres choix narratifs. Par ailleurs, le documentaire et les vidéos utilisées en formation seront un moyen de construire et transmettre la vision du syndicalisme portée par l'USS : un syndicalisme qui s'appuie sur les IRP, mais pour développer une approche de lutte et de classe.

Cette place centrale de l'image dans la diffusion des luttes est un enjeu essentiel pour l'ensemble des acteurs de conflits sociaux et politiques comme pour celles et ceux qui les documentent. La limitation du travail tant des journalistes que des documentaristes sur le terrain de luttes sociales constitue l'un des plus grands reculs pour la liberté de la presse, elle contraint déjà nombre de professionnelles à adapter leur pratique et pourrait même dissuader certaines de documenter des sujets jugés trop périlleux. À terme, ces attaques contre celles et ceux qui produisent des images pourraient même constituer un frein majeur au financement et à la diffusion de certains sujets, attaquant par-là l'essence même du débat démocratique. Ces tentatives pour limiter la production d'images non contrôlée sont une nouvelle preuve de leur efficacité sur le terrain des 
luttes, pour l'autonomie de celles et ceux qui sont bien souvent oubliés des récits journalistiques, de celles et ceux à qui l'on coupe le micro, toujours. En ce sens, l'ensemble de la démarche et des usages de l'image de l'USS porte une perspective radicale de renversement de l'ordre social.

\section{BIBLIOGRAPHIE}

Béroud S., Giraud B. et Yon, K. (2018) Sociologie politique du syndicalisme. Introduction à l'analyse sociologique des syndicats, Paris, Armand Colin.

Bovier F. et Fluckiger C. (2016) « René Vautier et le cinéma ouvrier : l'UPCB, une structure de production au service des « colonisés de l'intérieur » », Décadrages, [En ligne] https:// journals.openedition.org/decadrages/802.

Bret H. (2020) «Se dépenser et se préserver. Éboueurs et balayeurs du secteur public », Travail, genre et sociétés, vol. 43, $\mathrm{n}^{\circ} 1,2020$, p. 51-66.

Bué N., Delmas C. et Merlin J.-R. (2014) « Les dispositifs de formation syndicale sur la souffrance au travail » dans Ethuin N. et Yon K. (dir.), La Fabrique du sens syndical. La formation des représentants des salariés en France (1945-2010), Bellecombe-en-Bauges, Le Croquant.

Champagne P. (1984) «La manifestation. La production de l'événement politique », Actes de la recherche en sciences sociales, vol. 52-53, p. 19-41.

Champagne P. (1999) «Les médias et les risques », Actes du séminaire Risques collectifs et situations de crise, CNRS éditions, Paris.

Champagne P. (2011) «Le coup médiatique. Les journalistes font-ils l'événement? ", Société \& Représentations, $\mathrm{n}^{\circ} 32$, p. 25-43.

Corteel D. et Le Lay S. (2011) Les Travailleurs des déchets, Toulouse, Érès.

Depardon R. (1993) « Raymond Depardon. Pour une photographie des temps faibles », propos recueillis par A Rouillé, E. Hermange et V. Lavoie, La Recherche photographique, « Les Choses », $\mathrm{n}^{\circ} 15$, p. 80.

Fleckinger H. (2010) Carole Roussopoulos : Caméra militante. Luttes de libération des années 1970, Genève, Éditions MētisPresses.

Giraud B. (2009) Faire la grève. Les conditions d'appropriation de la grève dans les conflits du travail en France, Thèse de doctorat, Université Paris-1.

Giraud B., Yon K. et Béroud S. (2018) Sociologie politique du syndicalisme. Introduction à l'analyse sociologique des syndicats, Paris, Armand Colin.

Goffman E. (1973) La mise en scène de la vie quotidienne, la présentation de soi, Paris, Éditions de Minuit.

Graff S. (2011) " "Cinéma-vérité" ou "cinéma direct" : hasard terminologique ou paradigme théorique? ", Décadrages, https://journals.openedition.org/decadrages/215. 
Granjon F. (2001) L'Internet militant. Mouvement social et usages des réseaux télématiques, Paris, Apogée.

Heusch L. (1992) Naissance du cinéma documentaire sociologique en Europe, Grenade, Fonds Henri Storck.

Lagneau É. (2005) «Comment étudier la médiatisation des conflits? » dans Denis J.-M. (dir.), Le Conflit en grève? ? Paris, La Dispute.

Lagroye J. et Offerlé M. (dir.) (2011) Sociologie de l'institution, Paris, Belin.

Leal Ferreira L. (2004) « À propos de l'Enquête ouvrière de Karl Marx (1880) », Travailler, vol. 12, $\mathrm{n}^{\circ} 2$, p. 15-20.

Lioult J. (2004) À l'Enseigne du réel. Penser le documentaire, Aix-en-Provence, Presses universitaires de Provence.

Lynch É. (2013) « Détruire pour exister : les grèves du lait en France (1964, 1972 et 2009) », Politix, $\mathrm{n}^{\circ} 103$, p. 99-124.

Mariette A. (2011) « Pour une analyse des films de leur production à leur réception : Du "cinéma social" au cinéma comme lieu de mobilisations collectives », Politix, 1 (1), p. 47-68.

Marx K. [1880] (2004) « Enquête ouvrière ", Travailler, vol. 12, nº 2, p. 21-28.

Mercier A. (1994) «Mobilisation collective et limites de la médiatisation comme ressource » dans Fillieule O., Sociologie de la protestation. Les formes de l'action collective dans la France contemporaine, Paris, L'Harmattan.

Mercier A. (2018) « De l'événement au traitement événementiel des faits d'actualité », Hermès, La Revue, vol. 80, nº 1, p. 134-138.

Muel B. (2000) « Les riches heures du groupe Medvedkine (Besançon - Sochaux 1967-1974), Images documentaires 37/38, « Parole ouvrière ", p. 15-35.

Neveu E. (1999) « Médias, mouvements sociaux, espaces publics », Réseaux, vol. 17 nº 98, p. 17-85.

Neveu E. (2010) « Médias et protestation collective », dans Agrikoliansky É. et al., Penser les mouvements sociaux, La Découverte, coll. « Recherches », p. 245-264.

Nichols B. (1991) Representing Reality. Issues and Concepts in Documentary, Bloomington, Indiana University Press.

Offerlé M. (1998) Sociologie des groupes d'intérêt, Paris, Montchrestien.

Olivesi S. (2013) La Communication syndicale, Rennes, PUR.

Pelisse J. (2009) « Judiciarisation ou juridicisation? Usages et réappropriations du droit dans les conflits du travail », Politix, $n^{\circ} 86$, p. 73-9.

Perec G. (1989) L'Infra-ordinaire, Paris, Le Seuil.

Perron T. (1995) « "Le contrepoison est entre vos mains, camarades”. CGT et cinéma au début du siècle ", Le Mouvement social, p. 21-38.

Sinigaglia J. (2012) Artistes, intermittents, précaires en lutte. Retour sur une mobilisation paradoxale, Nancy, PUN.

Sirot S. (2002) La Grève en France : une histoire sociale (XIX $-\mathrm{XX}{ }^{e}$ siècle), Paris, Odile Jacob.

Collectif Vidéa (1976) «Vidéa : filmer les luttes, les vies, les rêves des femmes ", Cinéma d'aujourd'hui, nº 5/6: Cinéma militant, p. 147. 
Vautier R. (1978) « Cinémas de rupture : le point de vue de René Vautier », Écran, nº 65, janvier (rencontre organisée en novembre 1977 par « Cinéma politique »).

\section{NOTES}

1. Le film documentaire Grève au royaume des ordures a été coréalisé par les deux autrices de cet article. En tant que doctorante en sociologie en contrat avec l'Union syndicale, Lina Cardenas a filmé la plupart des images du documentaire alors qu'elle participait au piquet de grève. Jordane Burnot a, quant à elle, effectué le montage du film et réalisé les entretiens qui ponctuent le documentaire. Elle a également filmé les images tournées a posteriori.

2. Atelier roannais de construction textile.

3. Ordonnance de référé du 18 avril 2019.

4. Le collectif Vidéa est un collectif exclusivement féminin créé en 1974 par quatre jeunes réalisatrices (Anne-Marie Faure, Syn Guérin, Catherine Lahourcade et Isabelle Fraisse) dans une perspective féministe de documentation des luttes.

5. Bill Nichols propose de distinguer six modes du documentaire : le mode observation, le mode exposé, le mode poétique, le mode interactif-participatif, le mode réflexif, le mode performatif. Ces modes peuvent cohabiter dans un même film ou se succéder. Ces catégories vont déterminer un grand nombre de choix dans la production documentaire, de l'écriture, aux moyens mis en œuvre sur le tournage jusqu'au montage.

\section{RÉSUMÉS}

Ce travail entend apporter des pistes de réflexion afin de nourrir les travaux sur la construction iconographique des mobilisations collectives. Il s'agira plus particulièrement de retracer la pluralité d'usages des images lors de la grève des éboueurs·euses lyonnais·ses en avril 2019 soutenue par l'Union syndicale Solidaires (USS). Cet outil de représentation du conflit est investi par les grévistes et par les soutiens faisant ainsi des réseaux sociaux un espace de mobilisation en soi. L'image produite devient également un contrepoint médiatique essentiel pour rendre visibles les revendications des grévistes. De même, l'image va se déplacer dans les tribunaux lors de la judiciarisation du conflit par l'employeur pour appuyer les arguments de part et d'autre des parties. Pour finir, les militants s'approprient des images tournées pendant la mobilisation pour faire un documentaire et des supports de formation dans l'objectif de développer le syndicalisme porté par les militants de l'USS.

This work intends to provide lines of thought in order to feed the work on the iconographic construction of collective mobilizations. More specifically, it will retrace the plurality of uses of images during the strike of Lyon's garbage collectors in April 2019 supported by the Union Syndicale Solidaires (USS). This tool for representing the conflict is invested by the strikers and by the supporters, thus making social networks a space for mobilization in itself. The image produced becomes an essential media counterpoint to make the strikers' demands visible. In the same way, the image will move to the courts when the employer takes the dispute to court and will support the arguments on either side of the parties. Finally, the activists take ownership of 
the images shot during the mobilization to make a documentary and training materials with the aim of developing the trade unionism carried by the USS activists.

\section{INDEX}

Keywords : strike, documentary, social intervention film, medias, visual sociology, garbage collectors, trade unionism

Mots-clés : grève, documentaire, cinéma d'intervention social, médias, sociologie visuelle, éboueurs, syndicalisme

\section{AUTEURS}

\section{JORDANE BURNOT}

Jordane Burnot est réalisatrice de films documentaires. Elle s'intéresse particulièrement aux questions de mémoires, au monde ouvrier/du travail, à la thématique de l'exil et aux rapports de genre.

\section{LINA CARDENAS}

Lina Cardenas est doctorante en sociologie au LATTS à l'université Gustave Eiffel. Sa recherche doctorale porte sur la formation syndicale en France et particulièrement sur le cas de l'Union syndicale Solidaires. L'observation et la participation aux dispositifs de formation l'ont amenée à penser de nouveaux outils de formation, notamment à travers la création d'images lors de luttes syndicales. Ces productions sont désormais intégrées aux différents moyens de transmission de la mémoire militante de l'organisation. 